\title{
Non-functional urinary bladder paraganglioma with negative Ga-DOTA-NOC PET/CT uptake
}

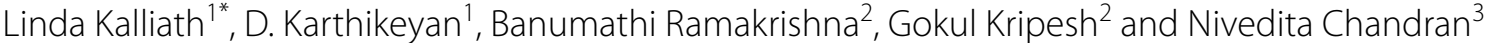

\begin{abstract}
Background: Paraganglioma of the urinary bladder is a very rare benign tumor that constitutes less than $0.5 \%$ of all bladder tumors. It can be functional and non-functional. Functional tumors present with symptoms of catecholamine excess, and non-functional tumors usually remain asymptomatic.

Case presentation: Here, we present a case of non-functional urinary bladder paraganglioma in a 36-year-old female who presented to us with a history of intermittent hematuria. The routine hematological and biochemical parameters were within normal limits. Diagnostic computed tomography (CT) revealed a hypervascular lesion in the urinary bladder dome with no significant uptake in gallium-68-labelled-1,4,7,10-tetraazacyclododecane-1,4,7,10tetraacetic acid peptides-Nal3-octreotide positron emission tomography (DOTA-NOC PET/CT). Histopathology and immunohistochemistry confirmed the diagnosis of paraganglioma.
\end{abstract}

Conclusion: We present this case because of the rare location of paraganglioma in the urinary bladder and being non-functional with low/absent somatostatin receptor expression.

Keywords: Paraganglioma, Urinary bladder, Non-functional, Computed tomography, DOTA-NOC PET/CT

\section{Background}

Urinary bladder paraganglioma is a very rare tumor that constitutes less than $0.5 \%$ of all bladder tumors. Usually, it behaves in a benign pattern. They can be functional and non-functional. Functional tumors present with the clinical manifestations of catecholamine excess, whereas non-functional tumors remain asymptomatic. The classical triad of bladder paraganglioma includes persistent hematuria, episodic hypertension, and post-micturition syncope. Diagnostic CT, scintigraphy, and advanced functional imaging such as somatostatin analog PET/ CT help in the preoperative evaluation of the lesion. In addition, histopathology and immunohistochemistry confirmed the diagnosis, especially in the case of

\footnotetext{
*Correspondence: lindakalliath@gmail.com

1 Department of Radiology, SRM Institutes for Medical Science,

Vadapalani, Chennai, India

Full list of author information is available at the end of the article
}

non-functional tumors. The most common differential is urothelial malignancy. Others include leiomyoma, hemangioma, and rhabdomyosarcoma.

\section{Case summary}

A 36-year-old female presented to the urology outpatient clinic with complaints of dysuria and intermittent hematuria with clots for 3 months. There were no history of headache, fainting, or palpitations. Her blood pressure on admission was $130 / 90 \mathrm{mmHg}$. There was no history of hypertension. The general examination did not reveal any significant abnormality. The routine hematological and biochemical parameters were within normal limits.

On ultrasound examination (Fig. 1), there was a welldefined heterogeneously hypoechoic mass lesion measuring $\sim 3.3 \times 2.1 \mathrm{~cm}$ in the right lateral wall of the dome of the urinary bladder with a focal area of calcification and significant internal vascularity. original author(s) and the source, provide a link to the Creative Commons licence, and indicate if changes were made. The images or other third party material in this article are included in the article's Creative Commons licence, unless indicated otherwise in a credit line to the material. If material is not included in the article's Creative Commons licence and your intended use is not permitted by statutory regulation or exceeds the permitted use, you will need to obtain permission directly from the copyright holder. To view a copy of this licence, visit http://creativecommons.org/licenses/by/4.0/. 


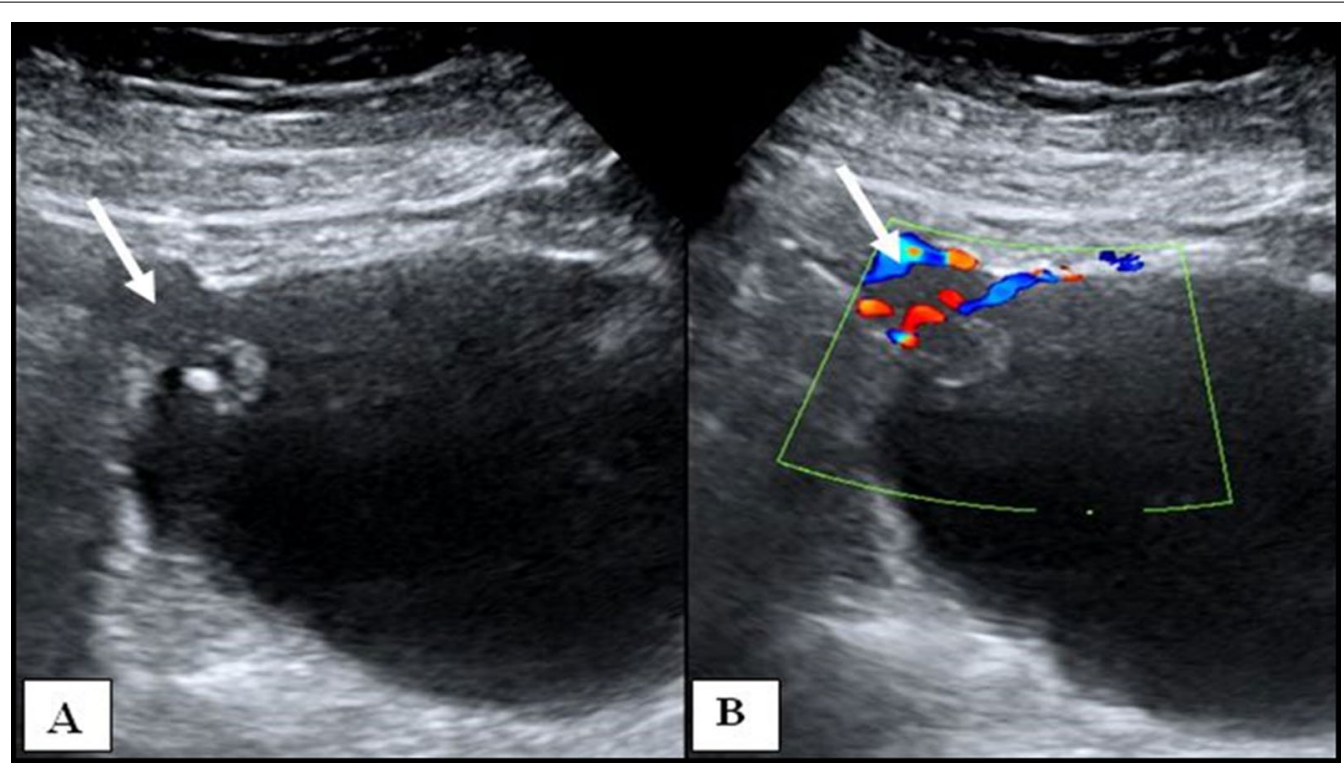

Fig. 1 A Grayscale ultrasound image of the urinary bladder shows a heterogeneously hypoechoic mass in the right lateral wall (arrow). B Color Doppler ultrasound image reveals significant internal vascularity within the lesion (arrow)

For further characterization, a contrast CT abdomen was done (Fig. 2). In the non-contrast study, there was an exo-endophytic lobulated mass lesion in the right anterolateral aspect of the dome of the urinary bladder with a focal punctate calcification seen. On contrast administration, intense arterial phase enhancement with feeding vessels (Fig. 3) from bilateral internal iliac arteries was seen. Persistent enhancement was noted in the venous phase without any evidence of washout. In view of hyperenhancing bladder wall lesion, differential diagnosis of urinary bladder paraganglioma and a less likely diagnosis of transitional cell carcinoma were made. DOTANOC PET/CT (Fig. 4) reveals enhancing mass lesion in the right lateral wall of the bladder with no significant uptake. No evidence of activity was seen elsewhere.

The patient underwent laparoscopic partial cystectomy, and the lesion was excised. There was no fluctuation in blood pressure during the surgery, and the procedure was uneventful. Macroscopic examination revealed a tan-brown polypoidal mass lesion (Fig. 5). On light microscopy, sections from the bladder mass revealed a submucosal neoplasm composed of monomorphous polygonal cells arranged in solid nests and intersected by delicate fibrovascular septae (Fig. 6). The tumor cells had stippled nuclear chromatin and moderate amounts of eosinophilic granular cytoplasm. Mitotic activity was inconspicuous. The tumor was found to involve the deep muscle fibers of the bladder wall. Immunohistochemistry (Fig. 7) revealed the tumor cells staining positive for synaptophysin and chromogranin, while negative for cytokeratin (AE1/AE3). Ki-67 proliferation index was 3\%, and a final diagnosis of paraganglioma was made. Postoperative follow-up revealed satisfactory recovery and no residual lesion.

\section{Discussion}

Urinary bladder paraganglioma is a very rare tumor that arises from chromaffin cells of the sympathetic nervous system in the bladder wall [1]. Urinary bladder paraganglioma constitutes less than $0.5 \%$ of all bladder tumors and $\sim 1 \%$ of all paragangliomas [2]. Among paragangliomas of the genitourinary tract, the urinary bladder is the most common site $(\sim 79.2 \%)$, which is followed by the urethra $(\sim 12.7 \%)$, renal pelvis $(\sim 4.9 \%)$, and ureter $(\sim 3.2 \%)$ [1]. Most of the bladder paragangliomas are seen in middle-aged adults and are common among females. Mostly, they have a benign clinical course, only 10\% turn to be malignant [3]. They can be functional or nonfunctional. Functional paragangliomas secrete excess catecholamines and will be symptomatic. Non-functional paraganglioma remains asymptomatic and is usually detected incidentally [4]. The typical presentation of bladder paraganglioma includes hematuria and symptoms induced by micturition due to increased catecholamine release, which includes hypertension, palpitation, and headache [5]. Non-functional bladder paraganglioma presents as painless hematuria or urinary tract obstruction [4]. The classical triad of bladder paraganglioma includes persistent hematuria, episodic hypertension, and post-micturition syncope, but this is rarely seen [3]. 

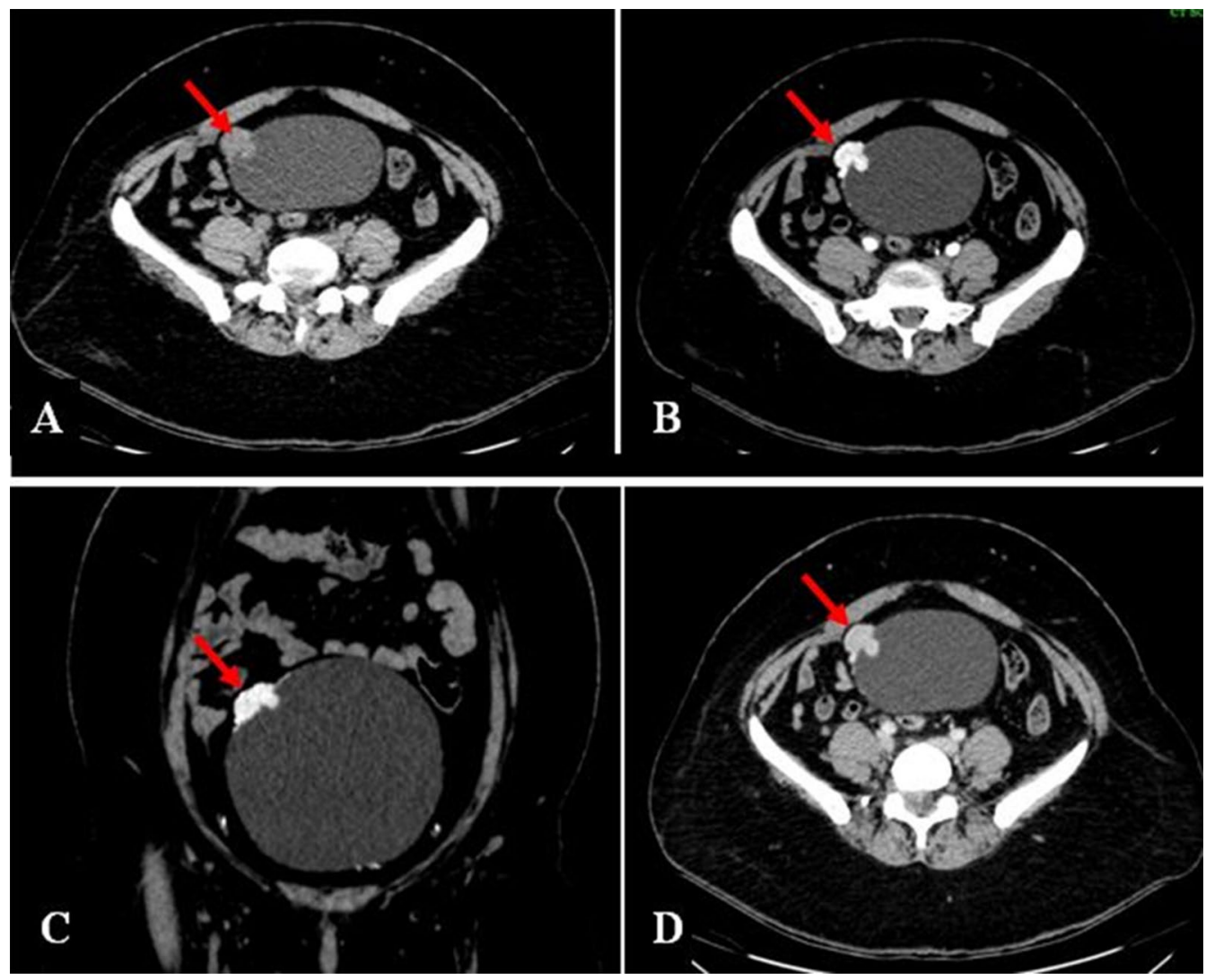

Fig. 2 A Non-contrast CT reveals exo-endophytic lobulated mass lesion (arrow) in the right lateral aspect of the dome of the urinary bladder. CECT abdomen arterial phase axial (B) and coronal reformatted (C) images show intense arterial enhancement of the lesion (arrow). Venous phase axial (D) images show persistent enhancement (arrow) with absent washout

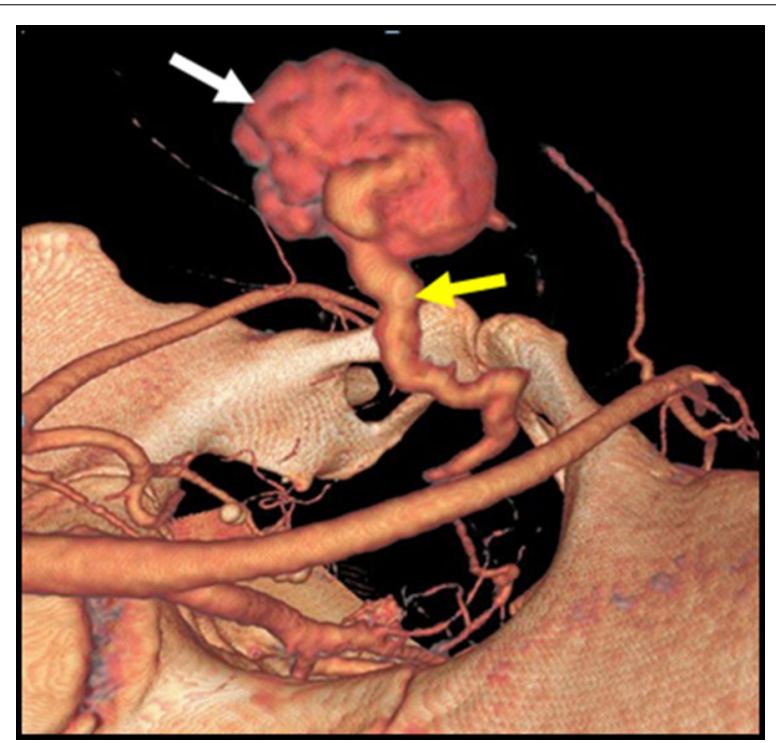

Fig. 3 3D volume-rendered CT image shows feeding vessels (yellow arrow) from the internal iliac artery to the highly vascular urinary bladder wall lesion (white arrow)
Even though sporadic, urinary bladder paraganglioma can occur familially or as part of syndromes like neurofibromatosis 1, von Hippel-Lindau syndrome, multiple endocrine neoplasias 2, and carney complex [2].

On imaging, bladder paraganglioma appears as a solid well-defined vascular lesion and is seen within the submucosal layer of the bladder [2]. Up to $40 \%$ are located in the dome and above the trigone of the bladder [6]. Nonfunctional bladder paraganglioma is commonly detected by ultrasound. On ultrasound, they appear as hypervascular echogenic mass and may have cystic foci within, representing areas of necrosis [3]. Paragangliomas are susceptible to degeneration and may show areas of necrosis, cystic changes, hemorrhage, and calcification [4]. On CT, it appears as soft tissue attenuation mass lesion, which shows homogeneous intense hyperenhancement on contrast study. It reflects the rich blood supply of paraganglioma. In the case of degeneration, heterogeneous enhancement is seen [1]. Areas of hemorrhage and calcification can also be seen [7]. On magnetic resonance imaging (MRI), the lesion shows multiple hyperintense foci interspersed with areas of signal voids, giving the 


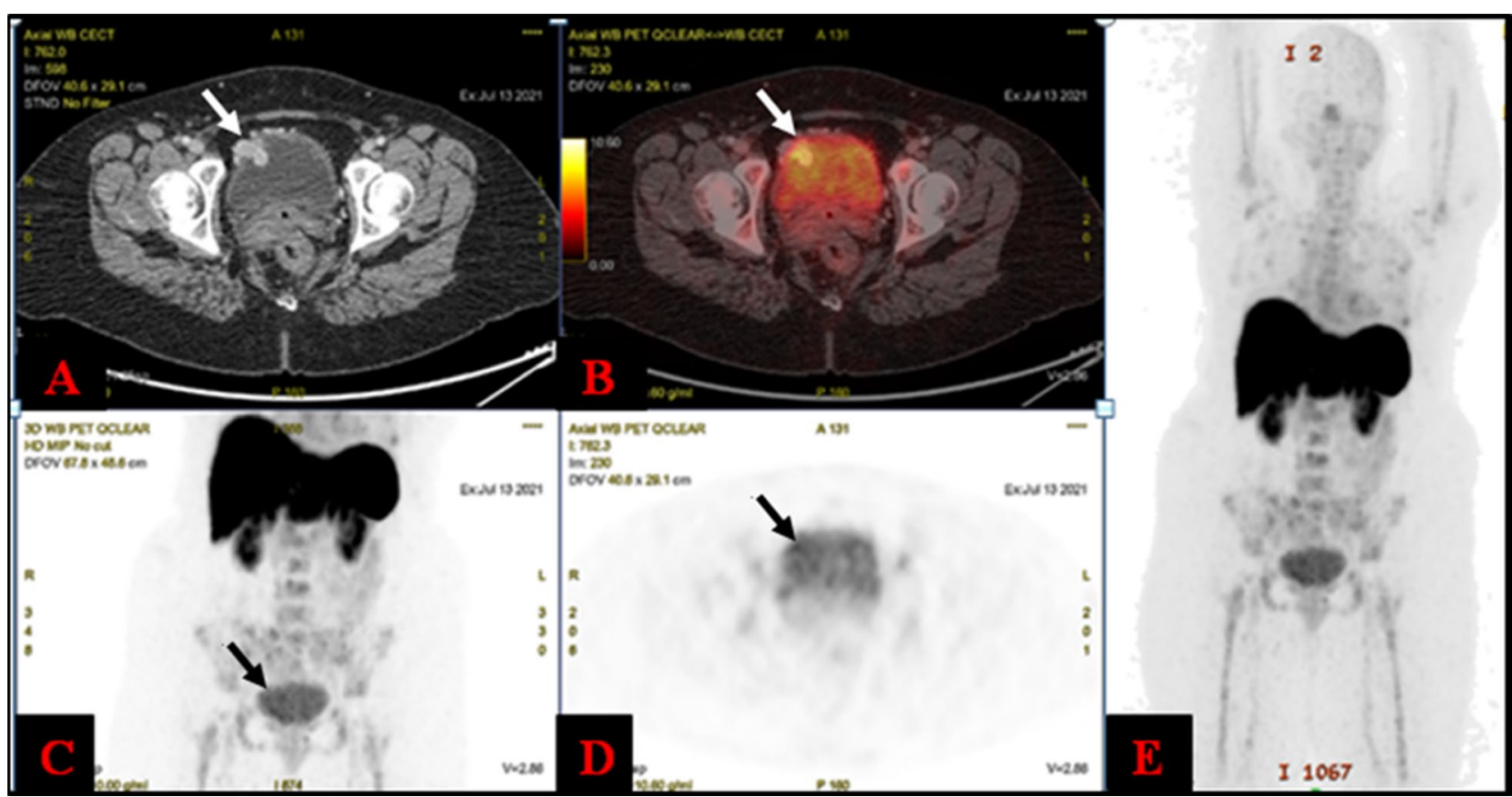

Fig. 4 On Ga DOTANOC PET/CT images (A, B) enhancing bladder mass lesion was seen (white arrow) but does not exhibit any increased tracer uptake. Corresponding maximum intensity projection images $(\mathbf{C}, \mathbf{D})$ reveal there was physiological tracer excretion and no significant uptake in the lesion (black arrow), and no somatostatin receptor-positive lesions elsewhere in the body (E)

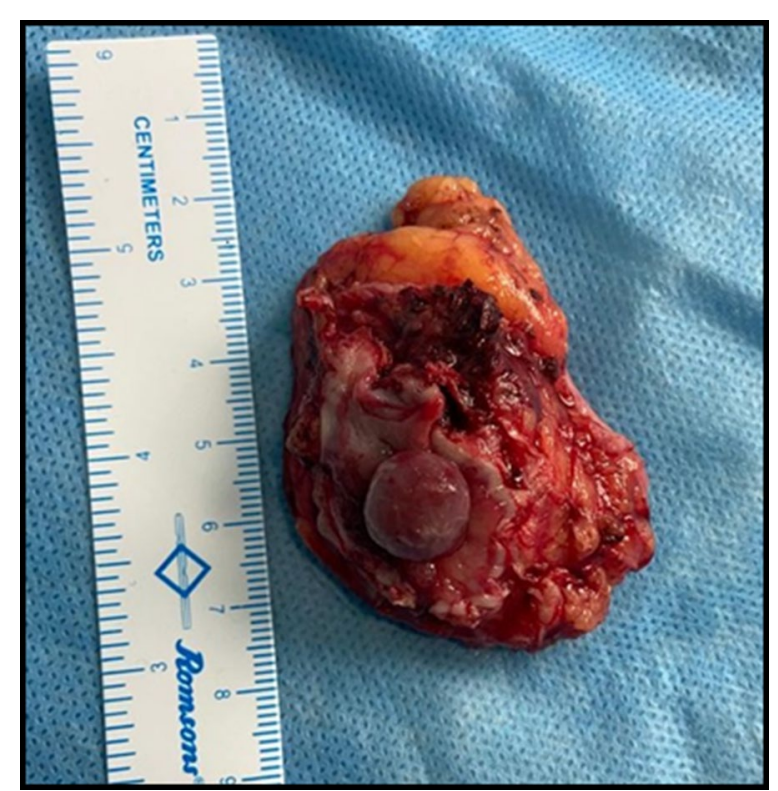

Fig. 5 Photograph of resected urinary bladder tumor shows a solid tan brown mass

classical salt and pepper appearance [1]. On T2-weighted imaging, the lesion demonstrates increased signal intensity due to the high vascularity and intracellular water content. It appears hyperintense on diffusion-weighted

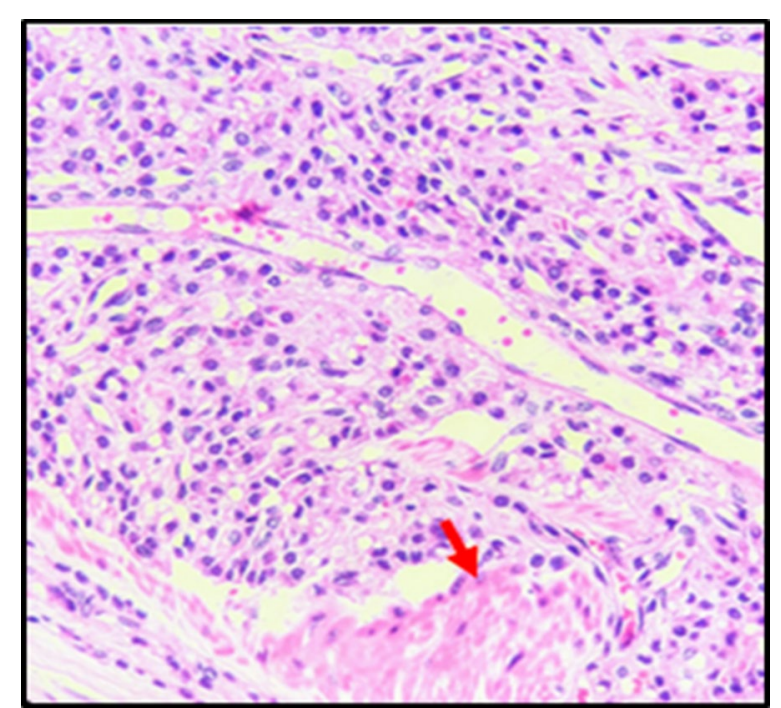

Fig. 6 Monomorphous polygonal cells arranged in solid nests, intersected by delicate fibrovascular septae and involving the deep muscle fibers (red arrow). H\&E 400x

images also [2]. Even though MRI is most sensitive for detection, M-iodobenzyl-guanidine (MIBG) study is considered highly specific for paraganglioma, since it provides functional information [7]. But its use is limited because of restricted availability and being highly 


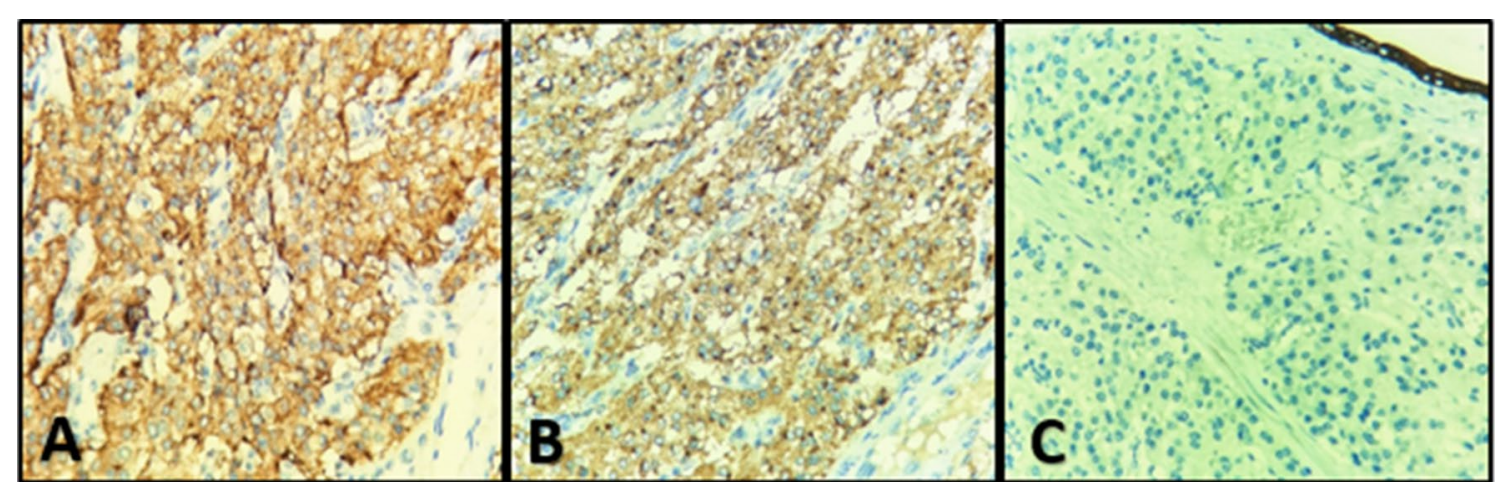

Fig. 7 Diffuse intracytoplasmic positivity (brown staining) in tumor cells for synaptophysin (A) and chromogranin (B) and negative staining (blue) for cytokeratin (C). Immunohistochemistry 400x

expensive [3]. Advanced functional imaging using somatostatin analogs has been used for early detection, staging, recurrence, and treatment assessment of these tumors. The new somatostatin analog PET molecules that have been developed include gallium-68-labeled1,4,7,10-tetraazacyclododecane-1,4,7,10-tetraacetic acid (DOTA)-peptides. PET CT with Ga DOTA-NOC has been proven to be highly sensitive and specific for neuroendocrine tumors than diagnostic CT and scintigraphy [8]. Negative tracer uptake in paragangliomas is seen related to small lesion size due to partial volume effect, alteration of receptor expression due to prior chemotherapy, or due to low or absent somatostatin receptor expression as in our case [9].

Functional paragangliomas secrete catecholamines, and a 24-h assessment of urinary vanillylmandelic acid helps in the diagnosis. Non-functional paragangliomas do not secrete excess catecholamines, and henceforth, preoperative diagnosis is difficult. In such cases, diagnosis depends on histopathology and immunohistochemistry [10]. Histologically paragangliomas are composed of tumor cells arranged in a zellballen pattern with abundant granular eosinophilic cytoplasm separated by vascularized fibrous septae [11]. As in our case, most of the paragangliomas are synaptophysin and chromogranin positive, but negative for cytokeratin, which is the appropriate way to diagnose a non-functional paraganglioma [4].

The most common differential of urinary bladder paraganglioma is urothelial bladder carcinoma. It is commonly seen in the bladder base $(\sim 80 \%)$ and typically occurs in elderly men with occupational exposure to carcinogens and with a history of smoking [2]. The lesion margins are not well delineated, show uneven enhancement, and can invade the bladder wall and other local organs [4]. Leiomyomas are the most common mesenchymal tumor of the bladder accounting for $\sim 35 \%$ of benign bladder tumors. It occurs commonly in young and middle-aged women. The lesion margins are well demarcated and show mild-to-moderate enhancement [4]. This lesion appears hypointense on T2-weighted imaging, which helps to differentiate from bladder paraganglioma. [2]. Rhabdomyosarcomas are the most common pediatric bladder tumor, with a high risk of malignancy, early metastasis, and poor prognosis. They present as grapelike lobulated mass with heterogeneous enhancement in the bladder base and mostly show local invasion [2]. Bladder hemangiomas can also be included in the differential of hypervascular lesions, but is common in the pediatric age group, and are commonly seen in the posterolateral wall of the bladder [2].

The treatment of choice is the surgical removal of the lesion, which includes transurethral resection and open/ laparoscopic cystectomy (partial/radical). In the case of functioning tumors, strict control of blood pressure by administering alpha-blocker in the pre- and intraoperative period is necessary to prevent hypertensive crisis [3]. Recurrent cases are treated by radiotherapy. Chemotherapy does not have an important role except for metastatic disease [7]. Long-term follow-up is necessary for paraganglioma to detect recurrence and metastasis [3].

\section{Conclusion}

Urinary bladder paraganglioma is a benign tumor mostly seen in adults presented with symptoms of catecholamine excess. The presence of a highly vascular hyperenhancing lesion with absent washout and elevated catecholamine metabolites combined with clinical manifestations should raise the suspicion of paraganglioma. In the case of non-functional paraganglioma, histopathology and immunohistochemistry aid in the diagnosis. We present this case because of its rare location in the urinary bladder and present a non-functional paraganglioma with low/absent somatostatin receptor expression. 


\section{Abbreviations}

CT: Computed tomography; DOTA-NOC PET/CT: Gallium-68 (68 Ga)-labelled1,4,7,10-tetraazacyclododecane-1,4,7,10-tetraacetic acid peptides-Nal3-octreotide positron emission tomography; PET: Positron emission tomography; MIBG: M-lodobenzyl-guanidine.

\section{Acknowledgements}

Not applicable.

\section{Authors' contributions}

Dr LK collected the data and wrote the manuscript; Dr DK helped in editing and supervising the paper; Dr BR and Dr GK are responsible for the curation of gross pathology, histological and immunohistochemistry images; and Dr NC provided the patient's clinical data and images. All authors have read and approved the final manuscript.

\section{Funding}

Not applicable.

\section{Availability of data and materials}

All data are available based on a reasonable request.

\section{Declarations}

Ethics approval and consent to participate

Not applicable.

\section{Consent for publication}

Formal informed consent for publication is obtained from the patient.

\section{Competing interests}

The authors declare that they have no competing interests.

\section{Author details}

${ }^{1}$ Department of Radiology, SRM Institutes for Medical Science, Vadapalani, Chennai, India. ${ }^{2}$ Department of Pathology, SRM Institutes for Medical Science, Vadapalani, Chennai, India. ${ }^{3}$ Department of Urology, SRM Institutes for Medical Science, Vadapalani, Chennai, India.

Received: 13 September 2021 Accepted: 22 November 2021

Published online: 26 November 2021

\section{References}

1. Dwethi G, Vikas C, Ramesh T (2019) Urinary bladder paraganglioma. Appl Radiol 48:48A-48C
2. Loveys FW, Pushpanathan C, Jackman S (2015) Urinary bladder paraganglioma: AIRP best cases in radiologic-pathologic correlation. Radiographics 35(5):1433-1438. https://doi.org/10.1148/rg.2015140303

3. Hanji AM, Rohan VS, Patel JJ, Tankshali RA (2012) Pheochromocytoma of the urinary bladder: a rare cause of severe hypertension. Saudi J Kidney Dis Transplant 23(4):813. https://doi.org/10.4103/1319-2442.98167

4. Qin J, Zhou G, Chen X (2020) Imaging manifestations of bladder paraganglioma. Ann Palliat Med 9(2):346-351. https://doi.org/10.21037/apm. 2020.03.09

5. Li S, Lui S, Li F, Yue Q, Huang X, Gong Q (2013) Unsuspected paraganglioma of the urinary bladder with intraoperative hypertensive crises: a case report. Exp Therap Med 6(4):1067-1069. https://doi.org/10.3892/ etm.2013.1242

6. Pahwa HS, Kumar A, Srivastava R, Rai A (2012) Unsuspected pheochromocytoma of the urinary bladder: reminder of an important clinical lesson. Case Rep. https://doi.org/10.1136/bcr-2012-006377

7. Sherwani P, Anand R, Narula MK, Siddiqui AA, Aggarwal S (2015) Concurrent nonfunctional paraganglioma of the retroperitoneum and urinary bladder: a case report with literature review. Indian J Radiol Imaging 25(2):198. https://doi.org/10.4103/0971-3026.155879

8. Haidar M, Shamseddine A, Panagiotidis E, Jreige M, Mukherji D, Assi R, Abousaid R, Ibrahim T, Haddad MM, Vinjamuri S (2017) The role of 68Ga-DOTA-NOC PET/CT in evaluating neuroendocrine tumors: realworld experience from two large neuroendocrine tumor centers. Nuclear Med Commun 38(2):170-177. https://doi.org/10.1097/MNM.0000000000 000623

9. Parra LA, Swanson SK, Salomao MA, Yang M (2020) Multifocal urinary bladder paragangliomas with negative 68Ga-DOTATATE uptake and positive 123I-MIBG uptake. Clin Nuclear Med 45(3):e156. https://doi.org/10. 1097/rlu.0000000000002853

10. Qiao B, Zhang B, Fu Z, Liu L, Liu C (2019) Non-functional paraganglioma of urinary bladder managed by transurethral resection. Int Braz J 45:910-915. https://doi.org/10.1590/s1677-5538.ibju.2018.0604

11. Spessoto LC, Vasilceac FA, Padilha TL, de Arruda PF, Gatti M, de Arruda JG, Pessutti D, da Silveira AT, Neto JD, Facio FN Jr (2016) Incidental diagnosis of nonfunctional bladder paraganglioma. Urol Case Rep 4:53-54. https:// doi.org/10.1016/j.eucr.2015.09.007

\section{Publisher's Note}

Springer Nature remains neutral with regard to jurisdictional claims in published maps and institutional affiliations.

\section{Submit your manuscript to a SpringerOpen ${ }^{\circ}$ journal and benefit from:}

- Convenient online submission

- Rigorous peer review

- Open access: articles freely available online

- High visibility within the field

- Retaining the copyright to your article

Submit your next manuscript at $\boldsymbol{\nabla}$ springeropen.com 